Published in final edited form as:

Clin Sci (Lond). 2018 February 28; 132(4): 437-447. doi:10.1042/CS20171457.

\title{
RAPAMYCIN ACTIVATES TGF RECEPTOR INDEPENDENTLY OF ITS LIGAND: IMPLICATIONS FOR ENDOTHELIAL DYSFUNCTION.
}

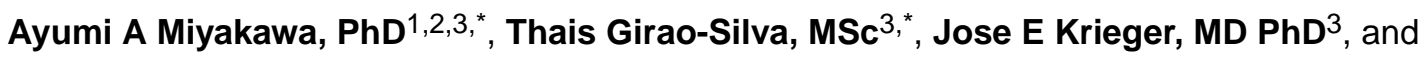 \\ Elazer R Edelman, MD, $\mathrm{PhD}^{1,2}$ \\ ${ }^{1}$ Cardiovascular Division, Department of Medicine, Brigham and Women's Hospital, Harvard \\ Medical School, Boston, MA 02115, USA; \\ ${ }^{2}$ Institute for Medical Engineering and Science, Massachusetts Institute of Technology, \\ Cambridge, MA 02139, USA; \\ ${ }^{3}$ Laboratory of Genetics and Molecular Cardiology, Heart Institute (InCor, HCFMUSP), Sao Paulo, \\ SP 054003, Brazil
}

\begin{abstract}
Rapamycin, the macrolide immunosuppressant and active pharmaceutic in drug-eluting stents (DES), has a well-recognized anti-proliferative action that involves inhibition of the mTOR pathway after binding to the cytosolic protein FKBP12. TGF receptor-type I (TGFRI) spontaneous activation is inhibited by the association with FKBP12. We hypothesized that rapamycin, in addition to inhibition of mTOR signaling, activates TGFRI independent of TGF $\beta$. Human umbilical vein endothelial cells (HUVEC) were treated with rapamycin (10nmoL/L) and/or TGF- $\beta$ RI kinase inhibitor (TGFRIi, 100nmoL/L) for 24 hours. Rapamycin induced SMAD phosphorylation (SMAD1, SMAD2 and SMAD5) and PAI-1 up-regulation, which was specifically abrogated by SMAD2 knockdown. TGFRIi efficiently blocked phosphorylation of SMAD2, but not SMAD1/5. Interestingly, the inhibitor did not alter cell proliferation arrest induced by rapamycin. Active TGF $\beta$ secretion was not affected by the treatment. Neutralizing TGF $\beta$ experiments did not influence SMAD2 phosphorylation or PAI-1 expression indicating that activation of this pathway is independent of the ligand. In addition, rapamycin induction of endothelial-to-mesenchymal transition (EndMT) was potentiated by IL-1 $\beta$, and efficiently blocked by TGFRIi. In vivo, the pro-thrombogenic effects of rapamycin and up-regulation of PAI-1 in murine carotid arteries were reduced by TGFRIi treatment. In conclusion, we provide evidence
\end{abstract}

Address for correspondence: Ayumi Aurea Miyakawa, Laboratory of Genetics and Molecular Cardiology, Heart Institute (InCor), University of Sao Paulo Medical School, Av. Dr. Eneas de Carvalho Aguiar, 44 - Bloco II, 10 andar, 05403-000 São Paulo - SP -

Brazil, ayumi.miyakawa@incor.usp.br.

These authors contributed equally to this work

AUTHOR CONTRIBUTION STATEMENT

Conception and design: AAM, ERE

Analysis and interpretation: AAM, TG-S, JEK, ERE

Data collection: AAM, TG-S

Writing the article: AAM, TG-S

Critical revision of the article: AAM, JEK, ERE

Statistical Analysis: AAM, TG-S

Obtained funding: AAM, JEK, ERE

Overall responsibility: AAM, ERE

DECLARATIONS OF INTEREST

None 
that rapamycin activates TGF receptor independent of its ligand TGF $\beta$, in concert with promotion of PAI-1 expression and changes in endothelial phenotype. These undesirable effects, the prothrombogenic state and activation of EndMT, are SMAD2-dependent and independent of the therapeutic rapamycin-induced cell proliferation arrest.

\section{Keywords}

endothelial cell; stent thrombosis; SMAD; TGF $\beta$ signaling; endothelial-to-mesenchymal transition

\section{INTRODUCTION}

Rapamycin is a macrolide immunosuppressant, also used in drug-eluting stents (DES) to suppress neointimal proliferation and prevent clinical restenosis. The DES effect is substantial but comes perhaps at the expense of thrombotic risk ${ }^{1-3}$. The anti-proliferative and anti-hyperplastic effects are classically ascribed to the binding by the drug and effective analogs to the FK506 binding protein 12 (FKBP12) ${ }^{4,5}$. Rapamycin-FKBP12 complex inhibits mammalian target of rapamycin (mTOR) signaling promoting cell cycle arrest ${ }^{4,5}$. Though vascular smooth muscle cells are affected first and foremost, there are also effects on endothelial cells, for example with the induction of endothelial tissue factor (TF) ${ }^{6,7}$ and plasminogen activator inhibitor- 1 (PAI-1) ${ }^{8-10}$ expression. These effects are cited as evidence for the prothrombogenic potential of the drug - this though is debated ${ }^{11}$ and, even if present, is not mechanistically defined.

PAI-1 is a member of serine protease inhibitor (serpin) family and a major inhibitor of fibrinolysis, the physiological breakdown of blood clots ${ }^{12,13}$. This protein expression. is induced by TGF $\beta$ receptor activation, specifically from receptor TGFRI/ALK5. FKBP12 interacts with TGF receptor-type I (TGFRI) and its spontaneous activation is kept in check by local complexation of FKBP12 $14-17$.

Thus, we hypothesized that rapamycin, in addition to its inhibition of mTOR, activates TGFRI in the absence of TGF- $\beta$, interfering with endothelial cell health. If true these data could explain the mode, nature and variability of rapamycin-induced effects on ECs and the vessel wall, and a possible means by which to deal with the thrombotic complications of DES.

\section{METHODS}

\section{Cell culture}

Human umbilical vein endothelial cells (HUVECs) were obtained from Invitrogen and maintained in EGM-2 media (PromoCell, Heidelber, Germany) supplemented with 2\% fetal bovine serum (FBS), $10 \mathrm{U} / \mathrm{mL}$ penicillin and $10 \mu \mathrm{g} / \mathrm{mL}$ streptomycin. Cells were used up to 8th passage. Before cell treatment, medium was changed to EGM-2 supplemented with $1 \%$ FBS for 24 hours. HUVECs were treated with $10 \mathrm{nmoL} / \mathrm{L}$ rapamycin (LC Laboratories, Woburn, MA) and/or $100 \mu \mathrm{moL} / \mathrm{L}$ TGF- $\beta$ RI kinase inhibitor (TGFRIi, ALK5 inhibitor, Calbiochem, Merck Millipore, Germany) for 24 hours. Control cells were treated with $0.1 \%$ DMSO vehicle. For long treatment experiments, cells were treated with $10 \mathrm{nmol} / \mathrm{L}$ 
rapamycin and/or $10 \mathrm{ng} / \mathrm{mL}$ IL-1 $\beta$ (PeproTech Inc., Rocky Hill, NJ) with or without TGF- $\beta$ RI kinase inhibitor $(100 \mu \mathrm{moL} / \mathrm{L})$ for 4 days. Medium were refreshed every day.

\section{SMADs silencing}

SMAD1, SMAD2 and SMAD5 silencing were performed using small interfering-RNA (siRNA) specific for each protein (sc-29483, sc-38374 and sc-38378, respectively). HUVEC were plated at $70 \%$ confluence and starved with EGM medium without serum for 16 hours. Lipofectamine 2000 (Invitrogen) was used to transfected cells with 40pmol of siRNA in Opti-MEM I medium (Invitrogen) for 6 hours. After this period, cells were washed and EGM-2 fresh medium was replaced. Treatment with rapamycin and/ or TGFR inhibitor was performed 24 hours after transfection.

\section{TGFb neutralizing antibody treatment}

HUVEC were previous incubated with $7,5 \mu \mathrm{g} / \mathrm{mL}$ TGF $\beta$ neutralizing antibody (R\&D) for 1 hour before rapamycin $(10 \mathrm{ng} / \mathrm{mL})$ treatment. TGF $\beta$ treatment $(5 \mathrm{ng} / \mathrm{mL})$ was used as positive control. Cells were lysed after 30 minutes or 24 hours for western blot evaluation of SMAD phosphorylation or PAI-1, respectively.

\section{Cell Activity}

HUVEC cultured medium was collected after 30 minutes or 24 hours and TGF- $\beta$ content was measured using TGF-beta 1 Emax ImmunoAssay System according to manufacturer's instructions (Promega, Madison, WI). All the samples were not submitted to acidification step in order to detect just active TGF $\beta 1$. Proliferation assay was performed by BrdU incorporation using ELISA colorimetric assay kit according to manufacturer's instructions (Roche Applied Science, Germany). Expression of critical proteins was determined by immunocytochemistry and Western blot.

\section{Immunocytochemistry}

Cells were fixed with $4 \%$ paraformaldehyde for 30 minutes. After permeabilization $(0.1 \%$ Nonidet P40 for 15 minutes) cells were incubated in blocking buffer (1\% BSA) for $1 \mathrm{~h}$. Primary antibodies for the following proteins were incubated overnight at $4{ }^{\circ} \mathrm{C}$ : VE-cadherin (Cell Signaling Technology, Inc., Danvers, MA), Phalloidin (Life Technologies, Molecular Probes, Inc., Eugene, OR), Calponin (EMD Millipore, Darmstadt, Germany), PECAM-1 (Abcam, Cambridge, MA) and SM22a (Abcam). Secondary antibodies Alexa488 or Alexa555 (Life Technologies, Molecular Probes, Inc.) were incubated for 2 hours and nucleus was stained with DAPI $10 \mu \mathrm{g} / \mu \mathrm{L}$. Immunocytochemistry were analyzed on Laser Scanning Microscope 510 (Carl Zeiss).

\section{Western blot}

Cells were lysed in SDS buffer (60mM TrisHCl pH 6.8, 5\% glycerol, 2\% SDS). Twenty-five to $50 \mu \mathrm{g}$ of cell lysates were run on SDS-polyacrylamide gels, transferred to PVDF membranes (Millipore, Billerica, MA), and incubated 1 hour in a blocking buffer (5\% Bovine Serum Albumin (BSA), $10 \mathrm{mM}$ Tris-HCl, pH 7.6, $150 \mathrm{mM} \mathrm{NaCl}$, and 0.1\% Tween 20). Each membrane was incubated overnight against a specific antibody: SMAD2 (Cell 
Signaling), pSMAD2 (Cell Signaling), SMAD1 (Cell Signaling), SMAD5 (Cell Signaling), pSMAD1/5 (Cell Signaling), PAI-1(Santa Cruz Inc., Dallas, TX), VE-cadherin (Cell Signaling), Calponin (Millipore), PECAM1 (Abcam), SM22a (Abcam). After incubation with peroxidase conjugated secondary antibodies, detection was performed with enhanced chemiluminescence reagents (GE Healthcare, Sweden). Protein levels of GAPDH (R\&D Systems, Minneapolis, MN) were used to normalize the results.

\section{Experimental animals and animal procedures}

C57BL/6 mice (20-30g) were treated with rapamycin ( $4 \mathrm{mg} / \mathrm{Kg}$ body weight/day) and/or TGF- $\beta$ RI kinase inhibitor ( $25 \mathrm{mg} / \mathrm{Kg}$ body weight/day) by manually restraining the animals and injecting the drug solutions intraperitoneally using a 25- or 27-gauge syringe. After 3 days, the carotid artery was collected for immunohistochemistry or subjected to photochemical thrombosis experiment.

All animal procedures followed institutional guidelines for the care and use of laboratory animals. This study protocol was approved by the committee of animal care of Massachusetts Institute of Technology (\# 0409-032-12).

\section{Immunohistochemistry}

The carotid segments were perfusion fixed in situ via cardiac puncture at $80 \mathrm{mmHg}$ with an initial infusion of saline with $14.8 \mathrm{mM} \mathrm{KCl}$, followed by $4 \%$ phosphate-buffered formalin. After $24-48 \mathrm{~h}$, the artery segments were dehydrated in graded ethanol baths, immersed in citrisolv, and embedded in paraplast (Oxford, St Louis, MO, USA). Transverse sections (3 $\mu \mathrm{m}$-thick) of the tissue were affixed onto slides positively charged. The slides were submitted to deparaffinization in xylenes and then rehydrated. Heat-induced antigen retrieval was done with Tris-EDTA buffer (pH 9.0) using pressure cooker. Slides were incubated in blocking buffer (Tris Buffer and 1\% casein) for 1h. PAI-1 (Santa Cruz) antibody was used at 1:50 dilution overnight at $4{ }^{\circ} \mathrm{C}$. After wash steps with Tris-buffer, slides were incubated with ALEXA 555 (Molecular Probes) for $2 \mathrm{~h}$ and nucleus stained with diamino-2-phenylindole (DAPI) at $10 \mu \mathrm{g} / \mu \mathrm{L}$. Immunohistochemistry were analyzed on Laser Scanning Microscope 510 (Carl Zeiss). Images were quantified by IMAGE J software.

\section{Photochemical Thrombosis Experiment}

Thrombosis in murine arteries followed by a protocol we previously described and validated ${ }^{18}$. Mice were anaesthetized with a ketamine-xylazine mixture and a Rose Bengal dye solution $(10 \mathrm{mg} / \mathrm{mL})$ was injected retro-orbitally into mice at a dosage of $50 \mathrm{mg} / \mathrm{kg}$. The carotid artery of the mouse was then exposed by careful dissection and a flow probe (Transonic, Inc., Ithaca, New York) was placed around the artery and a 2.0-mW laser with a wavelength of $543 \mathrm{~nm}$ (ThorLabs Inc., Newton, New Jersey) was applied to the artery. Blood flow was monitored until an occlusive thrombus was formed that stopped flow for at least 2 minutes.

\section{Statistical Analysis}

All data are expressed as mean \pm SEM. Comparison among groups were performed using two-way analysis of variance (ANOVA) followed by Tukey's post-hoc test for comparison. 
Student $t$ test was used for siRNA experiments, TGF-b release and photochemical thrombosis experiment. Values of $p<0.05$ were considered statistically significant.

\section{RESULTS}

\section{Rapamycin induces TGFRI activation and regulates PAI-1 expression via SMAD2}

We evaluated SMAD signaling to determine TGFRI activation by rapamycin. In endothelial cells, TGF $\beta$ activates SMAD2/3 signaling through ALK5/TGFRI and SMAD1/5/8 pathway through ALK1/TGFRI. Rapamycin was able to activate both types of receptors as indicated by phosphorylation of SMAD2 (Figure 1A) and SMAD1/5 (Figure 1B). The well demonstrated TGF-induced PAI-1 expression was also observed in rapamycin-treated cells and abrogated by SMAD2 knockdown. siRNA to SMAD1 and SMAD5 did not interfere with PAI-1 expression indicating that only SMAD2 signaling is activated in rapamycininduced PAI-1 expression (Figure 1C). The efficiency of SMADs silencing is represented in supplementary figure $1 \mathrm{~A}$.

A commercially available TGFRI inhibitor, blocking specifically SMAD2 phosphorylation (Figure 1A and 1B) and PAI-1 expression (Figures 1D), was used on the following experiments to understand the TGFRI activation by rapamycin better. SMAD2 activation by rapamycin is observed for at least 6 hours, and TGFRI inhibitor efficiently blocked its phosphorylation during this time (Supplementary Figure 1B). Indeed, induction of PAI-1 expression by rapamycin and its inhibition by TGFRI inhibitor can be observed even after 96 hours treatment (Figure Supplementary 1C).

Classically, rapamycin is known to inhibit mTOR activity and the downstream signaling such as $\mathrm{p} 70 \mathrm{~S} 6 \mathrm{~K}^{4,5}$. Interestingly, cell proliferation arrest induced by rapamycin was not altered by the TGFRI inhibitor (Figure 2A). Similarly, the reduction in p70S6K phosphorylation was maintained in the presence of TGFRI inhibitor (Figure 2B) and SMADs silencing (Figure 2C), suggesting that rapamycin activates two independent pathways to control cell proliferation and cell phenotype.

\section{Rapamycin induces TGFRI pathway independent of TGF- $\beta$}

To verify if TGFR activation occurs by increased release of active TGF $\beta$ induced by rapamycin, we performed an ELISA assay to measure TGF $\beta 1$ in the cell culture media. There was no difference in active TGF $\beta$ release in rapamycin-treated cells compared to control after 30 minutes (Figure 3A) and 24 hours (Figure 3B). Additionally, the increase of SMAD2 phosphorylation and PAI-1 expression induced by rapamycin was not affected by neutralizing TGF $\beta$ antibody (Figures 3C and 3D). The neutralizing effect of TGF $\beta$ antibody was demonstrated by reduction in SMAD2 phosphorylation and PAI-1 expression in TGF $\beta$ treated cells (Figure 3C). These data indicate rapamycin activates TGF receptor independently of TGF $\beta$.

\section{Rapamycin induces Endothelial-to-Mesenchymal Transition}

TGF $\beta$ has been implicated in endothelial-to-mesenchymal transition (EndMT) contributing to fibrosis ${ }^{19-21}$ and remodeling after injury ${ }^{22}$, therefore we examined EndMT in rapamycin 
activation of the TGF pathway. Because inflammatory co-stimulation has been described to efficiently induce EndMT ${ }^{23}$, we also performed the study in the presence of IL-1 $\beta$. EndMT is characterized by loss of endothelial markers and increased expression of mesenchymal markers ${ }^{19,24}$. Here we evaluated VE-Cadherin and PECAM-1 as endothelial markers and SM22 and calponin as mesenchymal markers. Although rapamycin has shown a small decrease in VE-Cadherin and PECAM1, it increased the mesenchymal marker SM22 and changed actin fiber pattern from cortical to fiber stress characteristic of mesenchymal and smooth muscle cells (Figure 4). In the presence of IL-1 $\beta$, the induction of EndMT by rapamycin was potentiated showing more pronounced decrease in VE-Cadherin and PECAM- 1 than in rapamycin-only treated cells. Additionally, rapamycin and IL- $1 \beta$ treated cells showed induction of calponin expression (Figure 4B and 4F), which was not observed by the treatment of IL- $1 \beta$ alone (supplementary figure 1C). The TGFRIi reverted mesenchymal marker expression in rapamycin and rapamycin combined with IL- $1 \beta$ treated cells but did not prevent the fiber stress pattern of actin organization.

\section{Blockage of TGFRI diminishes rapamycin-induced PAI-1 expression and prevents thrombogenicity in vivo}

Mice treated with rapamycin exhibited increased carotid arterial PAI-1 expression, which was attenuated by TGFRI inhibitor (Figure 5A and 5B). Similarly, vessels expressing more PAI-1 were more thrombogenic than vessels with less PAI-1 (Figure 5C and 5D), demonstrating that TGFRI inhibition prevents the thrombogenicity induced by rapamycin through PAI-1 downregulation.

\section{DISCUSSION}

Rapamycin effects are manifest as a chemotherapeutic agent and when presented in a local sustained and controlled manner as an inhibitor of local proliferative vascular disease. Use in the latter case also comes with the price of increased thrombogenicity and it has been proposed that this arises from loss of an intact endothelial barrier ${ }^{8,25}$. The drug might directly inhibit restoration of a denuded endothelium by impairing endothelial barrier function ${ }^{26,27}$ Rapamycin increases intracellular calcium which activates protein kinase $\mathrm{C}-\mathrm{a}$, promoting disruption of p120 catenin-VE cadherin and VE-cadherin degradation. ${ }^{27}$

We wondered if the effects of rapamycin were even more direct. Rapamycin inhibits mTOR complexation through binding to FKBP12 $2^{4,5}$. While inhibition of mTORC1 occurs immediately after binding of rapamycin to FKBP12, blockage of mTORC2 needs prolonged exposure of cells to rapamycin ${ }^{28,29}$. FKBP12 is a ubiquitous protein and its interaction with TGFRI inhibits basal signaling of the growth factor receptor ${ }^{14,16,17}$. Fibroblasts from FKBP12 knockout mice showed enhanced activity of TGFRI and inhibition of cell cycle ${ }^{17}$. As FKBP12 is a common component between TGF and rapamycin signaling, we asked if rapamycin could activate TGFRI by FKBP12 displacement from the receptor and by this means alter endothelial phenotype even without the TGF $\beta$ ligand.

The present work demonstrated that rapamycin phosphorylates SMAD1/5 and SMAD2 in a time-dependent manner in concert with the activation of TGFRI. In addition, rapamycin increases PAI-1 expression and induces EndMT similar to effects described for TGF $\beta$. 
Although SMAD 1, 2 and 5 are activated by rapamycin, only SMAD2 seems to contribute to changes of endothelial phenotype. The implications of activation of SMAD1/5 by rapamycin should be further investigated.

Others have shown effects of rapamycin on PAI- $1^{8-10}$, however the mechanism underlining this process remains unclear. To our knowledge, the present work is the first to demonstration that rapamycin induces PAI-1 expression by TGFRI activation in endothelial cells independently of TGF- $\beta$. In contrast, others have shown that rapamycin activates the TGF $\beta$ pathway by increasing expression and/or activation of TGF $\beta^{30,31}$. In rat mesangial cells, rapamycin activates latent TGF $\beta$ and increases PSMAD2 and PAI- $1^{31}$. The increased oxidative stress after 30 minutes of rapamycin treatment resulted in secretion of active TGF$\beta^{31}$. The difference of action can be explained by differences in regulation between cell types: depletion of FKBP12 in mesangial cell reduces SMAD2 phosphorylation instead of increases as demonstrated in endothelial cell type.

We are suggesting that rapamycin can activate TGFRI by FKBP12 displacement from the receptor. Indirect action on TGF pathway can also occur, as regulation of accessories receptor like $\beta$ glycan, endoglin, SARA (SMAD anchor for receptor activation) and SMAD7 ${ }^{32-34}$. Our in vivo experiments corroborated activation of TGF pathway by rapamycin, where the increase of PAI-1 expression after drug treatment correlates with increased thrombogenicity and TGFR inhibitor reduces this effect (Figure 5).

We also show that rapamycin can induce EndMT and affect locally the health of endothelial cells adding further to understanding the endothelial toxicity of rapamycin. EndMT, a process where endothelial cells lose their specific markers and morphology and acquire mesenchymal cell-like characteristics, has been implicated in heart valve mesenchyme formation ${ }^{35}$, cardiac and renal chronic fibrosis ${ }^{19-21}$ and vascular remodeling ${ }^{22}$. The most described regulator of EndMT is TGF $\beta$ and we demonstrate that rapamycin induces expression of mesenchymal markers in endothelial cells through activation of TGFRI. EndMT induced by rapamycin showed some aspects distinct from the process induced by TGF $\beta$. While both TGF $\beta$ and rapamycin induced the expression of mesenchymal markers, rapamycin treatment had a slight reduction in endothelial markers. Interestingly, TGFRI inhibitor reverted mesenchymal marker expression but did not prevent the fiber stress pattern of actin. This may be to the long term effect of rapamycin on inhibition of mTORC2 ${ }^{28}$. This complex is involved in actin cytoskeleton modulation through regulation of F-actin and actin-related proteins ${ }^{36}$. Though rapamycin does not recapitulate exactly the EndMT process, it is clear that rapamycin drives the endothelial cells to an activated state.

Normal endothelial function is essential for retention of vascular health, inhibiting thrombus formation, inflammation and smooth muscle cell proliferation. The incomplete reendothelialization of stent strut surfaces is a recognized substrate for stent thrombosis, and DES are thought to delay endothelial recovery allowing for persistent fibrin build up around the stent sturts ${ }^{37,38}$. We provide evidence that endothelial cells in the presence of rapamycin are dysfunctional and thrombogenic, suggesting that the thrombogenicity of DES is not only from loss of the complete endothelial monolayer but also from a prothrombotic endothelial cell phenotype. We suggest that rapamycin may act by two distinct pathways - one that 
inhibits mTOR and leads to cell cycle arrest, and the other that activates SMAD2 and increases PAI-1 expression and EndMT (Supplementary Figure 2).

Overall, this study shows that rapamycin activates the TGF $\beta$ pathway and modify endothelial phenotype to a more thrombotic state independent of TGF $\beta$. Understanding the impact of DES on endothelial function is essential to find new approaches that prevent restenosis and maintain the vascular health.

\title{
PERSPECTIVES
}

i. Rapamycin has a well-recognized anti-proliferative action that involves inhibition of the mTOR pathway after binding to the cytosolic protein FKBP12. TGFRI spontaneous activation is inhibited by the association with FKBP12. We hypothesized that rapamycin, when interacting with FKBP12, allows TGFRI activation independently of TGF $\beta$.

ii. Our findings have critical basic and applied implications. We demonstrated that rapamycin activates TGF receptor independent of its ligand TGF- $\beta$, promotes PAI-1 expression and induces changes in endothelial phenotype. Such profound effects on signaling of ligand-independent binding are in and of itself fascinating. We further showed that these effects were associated with endothelial activation and a thrombogenic state in culture and in vivo, that are SMAD 2-dependent and independent of the therapeutic rapamycin-induced cell proliferation arrest.

iii. These findings may then explain the variability in disparate findings in the past, the mechanism of stent thrombosis and a means of obviating these complications in practice.

\section{Supplementary Material}

Refer to Web version on PubMed Central for supplementary material.

\section{ACKNOWLEDGMENTS}

We thank Melissa St. Pierre for performing the surgical procedures of the photochemical thrombosis experiments. FUNDING INFORMATION

AAM was supported by the J.P. Lemann Foundation as a Jorge Paulo Lemann Harvard Medical School Cardiovascular Fellow at Brigham \& Women's Hospital. TG-S is recipient of fellowship from Sao Paulo Research Foundation (FAPESP 2014/06844-9). JEK is supported in part by grants from Sao Paulo Research Foundation (FAPESP 2013/17368-0) and ERE is supported in part by a grant from the US National Institutes of Health (R01 GM 49039).

\section{ABBREVIATIONS LIST}

\author{
DES drug-eluting stents \\ EndMT endothelial-to-mesenchymal transition \\ FKBP12 FK506 binding protein 12
}




$\begin{array}{ll}\text { HUVEC } & \text { Human umbilical vein endothelial cells } \\ \text { mTOR } & \text { mammalian target of rapamycin } \\ \text { PAI-1 } & \text { plasminogen activator inhibitor-1 } \\ \text { PECAM-1 } & \text { platelet/endotelial cell adhesion molecule 1 } \\ \text { TF } & \text { tissue fator } \\ \text { TGFRI } & \text { TGF receptor-type I } \\ \text { TGFRIi } & \text { TGF- } \beta \text { RI kinase inhibitor }\end{array}$

\section{REFERENCES}

1. Iakovou I et al. Incidence, predictors, and outcome of thrombosis after successful implantation of drug-eluting stents. Jama (2005). doi:10.1001/jama.293.17.2126

2. Kuchulakanti PK et al. Correlates and long-term outcomes of angiographically proven stent thrombosis with sirolimus- and paclitaxel-eluting stents. Circulation (2006). doi:10.1161/ CIRCULATIONAHA.105.600155

3. Daemen Joost; Wenaweser Peter; Tsuchida Keiichi; Abrecht Linda; Vaina Sophia; Morger Cyrill; Kukreja Neville; Jüni Peter; Sianos Georgios; Hellige Gerrit; van Domburg Ron T; Hess Otto M; Boersma Eric; Meier Bernhard; Windecker PW Early and late coronary stent thrombosis of sirolimus-eluting and paclitaxel-eluting stents in routine clinical practice: data from a large twoinstitutional cohort study. The Lancet, >2007, Vol.369(9562), pp.667-678 (2007). doi:10.1016/ S0140-6736(07)60314-6

4. Dumont FJ \& Su Q Mechanism of action of the immunosuppressant rapamycin. Life Sciences (1995). doi:10.1016/0024-3205(95)02233-3

5. Ballou LM \& Lin RZ Rapamycin and mTOR kinase inhibitors. J. Chem. Biol (2008). doi:10.1007/ s12154-008-0003-5

6. Camici GG et al. Rapamycin promotes arterial thrombosis in vivo: implications for everolimus and zotarolimus eluting stents. 236-242 (2010). doi:10.1093/eurheartj/ehp259

7. Steffel J et al. Rapamycin, but not FK-506, increases endothelial tissue factor expression: Implications for drug-eluting stent design. Circulation (2005). doi:10.1161/CIRCULATIONAHA. 105.569129

8. Muldowney JAS et al. Antiproliferative agents alter vascular plasminogen activator inhibitor-1 expression: A potential prothrombotic mechanism of drug-eluting stents. Arterioscler. Thromb. Vasc. Biol (2007). doi:10.1161/01.ATV.0000254677.12861.b8

9. Ota $\mathrm{H}$ et al. Sirolimus and everolimus induce endothelial cellular senescence via sirtuin 1 downregulation: therapeutic implication of cilostazol after drug-eluting stent implantation. J. Am. Coll. Cardiol (2009). doi:10.1016/j.jacc.2009.01.072

10. Ma Q et al. Rapamycin Affects Tissue Plasminogen Activator and Plasminogen Activator Inhibitor 1 Expression: A Potential Prothrombotic Mechanism of Drug-Eluting Stents. (2012). doi: $10.1177 / 0003319711418219$

11. Kolandaivelu K et al. Stent thrombogenicity early in high-risk interventional settings is driven by stent design and deployment and protected by polymer-drug coatings. Circulation (2011). doi: 10.1161/CIRCULATIONAHA.110.003210

12. Binder BR et al. Plasminogen activator inhibitor 1: physiological and pathophysiological roles. News Physiol. Sci 17, 56-61 (2002). [PubMed: 11909993]

13. Gils A \& Declerck PJ The structural basis for the pathophysiological relevance of PAI-1 in cardiovascular diseases and the development of potential PAI-1 inhibitors. Thromb. Haemost 91, 425-437 (2004). [PubMed: 14983217] 
14. Okadome T Characterization of the Interaction of FKBP12 with the Transforming Growth Factorbeta Type I Receptor in Vivo. J. Biol. Chem (1996). doi:10.1074/jbc.271.36.21687

15. Wang $T$ et al. The immunophilin FKBP12 functions as a common inhibitor of the TGF $\beta$ family type I receptors. Cell 86, 435-444 (1996). [PubMed: 8756725]

16. Chen Y \& Liu F Mechanism of TGF $\beta$ receptor inhibition by FKBP12. 16, 3866-3876 (1997).

17. Aghdasi B et al. FKBP12, the 12-kDa FK506-binding protein, is a physiologic regulator of the cell cycle. Proc. Natl. Acad. Sci. U. S. A (2001). doi:10.1073/pnas.041614198

18. Baker AB et al. Heparanase regulates thrombosis in vascular injury and stent-induced flow disturbance. J. Am. Coll. Cardiol (2012). doi:10.1016/j.jacc.2011.11.057

19. Zeisberg EM et al. Endothelial-to-mesenchymal transition contributes to cardiac fibrosis. Nat. Med (2007). doi:10.1038/nm1613

20. Zeisberg EM, Potenta SE, Sugimoto H, Zeisberg M \& Kalluri R Fibroblasts in Kidney Fibrosis Emerge via Endothelial-to-Mesenchymal Transition. J. Am. Soc. Nephrol (2008). doi:10.1681/ ASN.2008050513

21. Piera-Velazquez S, Li Z \& Jimenez SA Role of endothelial-mesenchymal transition (EndoMT) in the pathogenesis of fibrotic disorders. American Journal of Pathology (2011). doi:10.1016/j.ajpath. 2011.06.001

22. Cooley BC et al. TGF- $\beta$ signaling mediates endothelial to mesenchymal transition (EndMT) during vein graft remodeling. Sci. Transl. Med (2014). doi:10.1126/scitranslmed.3006927

23. Maleszewska $M$ et al. IL-1 $\beta$ and TGF $\beta 2$ synergistically induce endothelial to mesenchymal transition in an NFrB-dependent manner. Immunobiology (2013). doi:10.1016/j.imbio. 2012.05.026

24. Arciniegas E, Frid MG, Douglas IS \& Stenmark KR Perspectives on endothelial-to-mesenchymal transition: potential contribution to vascular remodeling in chronic pulmonary hypertension. Am J Physiol Lung Cell Mol Physiol 293, L1-8 (2007). [PubMed: 17384082]

25. Nakazawa G et al. Delayed Arterial Healing and Increased Late Stent Thrombosis at Culprit Sites After Drug-Eluting Stent Placement for Acute Myocardial Infarction Patients An Autopsy Study. (2008). doi:10.1161/CIRCULATIONAHA.107.762047

26. Inoue $\mathrm{T}$ et al. Vascular inflammation and repair: Implications for re-endothelialization, restenosis, and stent thrombosis. JACC Cardiovasc. Interv 4, 1057-1066 (2011). [PubMed: 22017929]

27. Habib A et al. Sirolimus-FKBP12.6 impairs endothelial barrier function through protein kinase C-?? activation and disruption of the p120-vascular endothelial cadherin interaction. Arterioscler. Thromb. Vasc. Biol (2013). doi:10.1161/ATVBAHA.113.301659

28. Sarbassov DD et al. Prolonged Rapamycin Treatment Inhibits mTORC2 Assembly and Akt / PKB. 159-168 (2006). doi:10.1016/j.molcel.2006.03.029

29. Barilli A et al. In human endothelial cells rapamycin causes mTORC2 inhibition and impairs cell viability and function. 563-571 (2017). doi:10.1093/cvr/cvn024

30. City SL, Organ LS \& Services T Sirolimus increases transforming growth factor- b 1 expression and potentiates chronic cyclosporine nephrotoxicity. 65, 1262-1271 (2004).

31. Osman B et al. Rapamycin induces the TGF $\beta 1$ / Smad signaling cascade in renal mesangial cells upstream of mTOR. Cell. Signal 21, 1806-1817 (2009). [PubMed: 19666112]

32. Huang F \& Chen Y Regulation of TGF- b receptor activity. 1-10 (2012).

33. Tsukazaki T, Chiang TA, Davison AF, Attisano L \& Wrana JL SARA, a FYVE domain protein that recruits Smad 2 to the TGF $\beta$ receptor. Cell 95, 779-791 (1998). [PubMed: 9865696]

34. Runyan CE, Schnaper HW \& Poncelet AC The role of internalization in transforming growth factor $\beta 1$-induced Smad 2 association with Smad anchor for receptor activation (SARA) and Smad2-dependent signaling in human mesangial cells. J. Biol. Chem (2005). doi:10.1074/ jbc.M407939200

35. Markwald RR, Fitzharris TP \& Manasek FJ Structural development of endocardial cushions. Am. J. Anat (1977). doi:10.1002/aja.1001480108

36. Oh WJ \& Jacinto E mTOR complex 2 signaling and functions. 2305-2316 (2011). doi:10.4161/cc. 10.14.16586 
37. Joner $\mathrm{M}$ et al. Endothelial Cell Recovery Between Comparator Polymer-Based Drug-Eluting Stents. J. Am. Coll. Cardiol (2008). doi:10.1016/j.jacc.2008.04.030

38. Finn AV et al. Vascular Responses to Drug Eluting Stents Importance of Delayed Healing. (2007). doi:10.1161/ATVBAHA.107.144220 
A

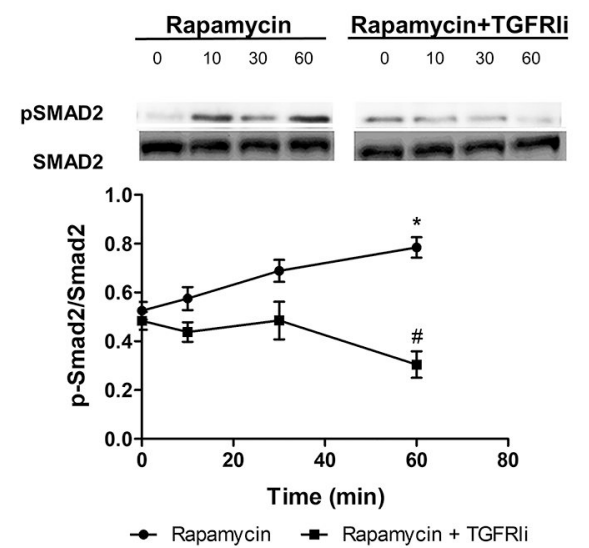

C

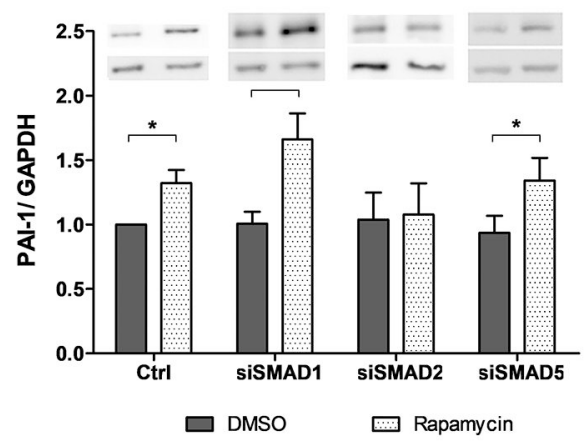

B

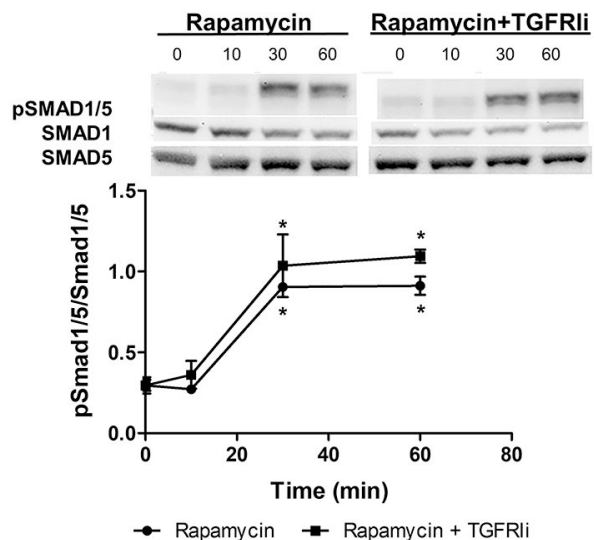

D

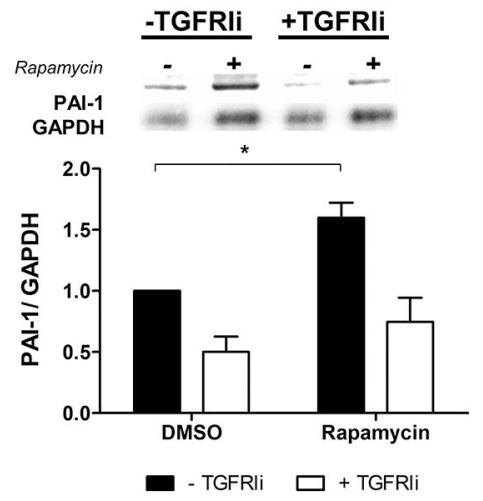

Figure 1.

SMAD activation and PAI-1 expression by rapamycin in endothelial cells. Time-course of (A) SMAD-2 phosphorylation and (B) SMAD1/5 phosphorylation by rapamycin in the absence $(\mathbf{O})$ or presence of TGFRI inhibitor (TGFRIi) $(\boldsymbol{\Delta})$. Each point represents mean \pm SEM of 4 independent experiments. * indicates $p<0.05$ compared to time 0 of each treatment and \# indicates $\mathrm{p}<0.05$ of rapamycin $(60 \mathrm{~min})$ vs. rapamycin + TGFRIi (60 min). (C) PAI-1 protein expression induced by rapamycin in endothelial cells knockdown for SMAD1, SMAD2 and SMAD5. Each bar represents mean \pm SEM of 6-7 independent experiments. * indicates $\mathrm{p}<0.05$. (D) PAI-1 protein expression induced by rapamycin in the presence of TGFRIi for 24 hours. Each bar represents mean \pm SEM of 3-5 independent experiments. Black bars represent cells without TGFRIi and white bars represent cells with TGFRIi. * indicates $\mathrm{p}<0.05$. 
A

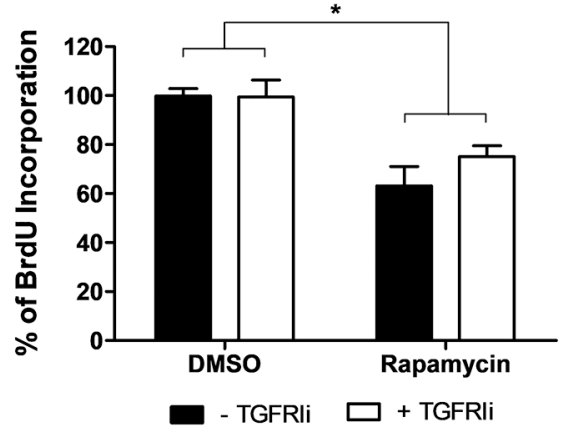

B

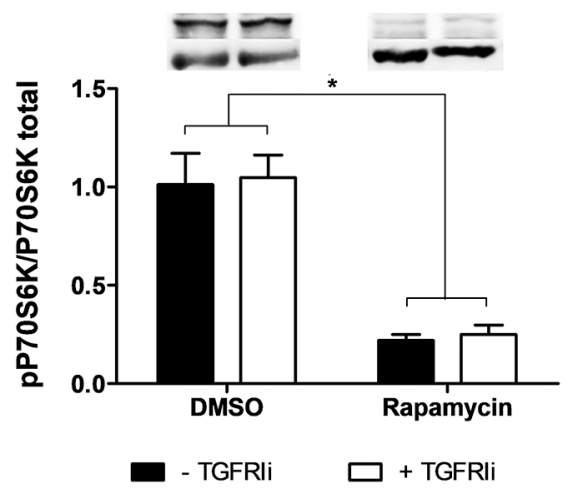

C

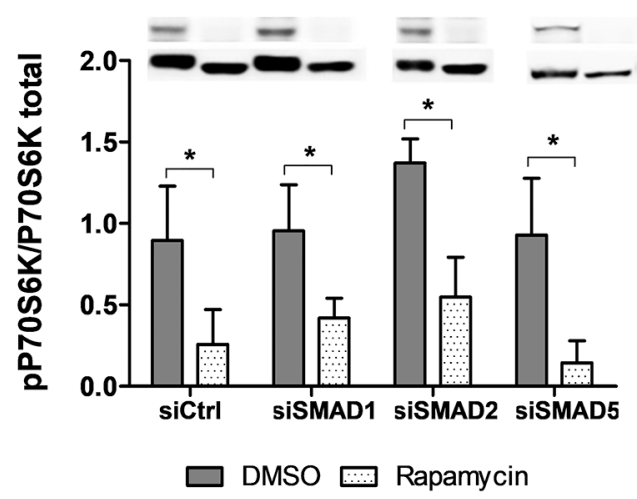

Figure 2.

Cell proliferation arrest induced by rapamycin is not altered by TGFRI inhibitor or SMAD knockdown. (A) Rapamycin inhibits cell proliferation analyzed by BrdU incorporation even in the presence of TGFRI inhibitor for 24 hours. Control cells without TGFRIi were considered $100 \%$ of BrdU incorporation. p70S6K phosphorylation expression after rapamycin treatment in the presence of (B) TGFRI inhibitor or (C) knockdown SMADs. Each bar represents mean \pm SEM of 3-5 independent experiments. * indicates $p<0.05$. 
A

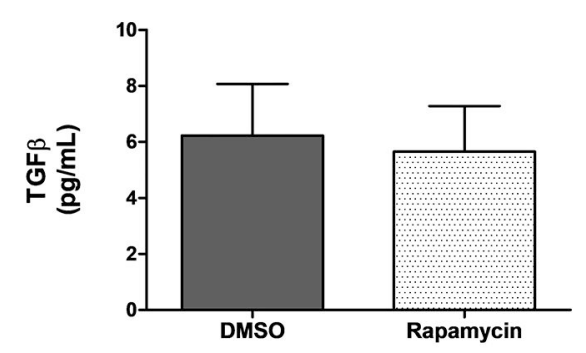

C

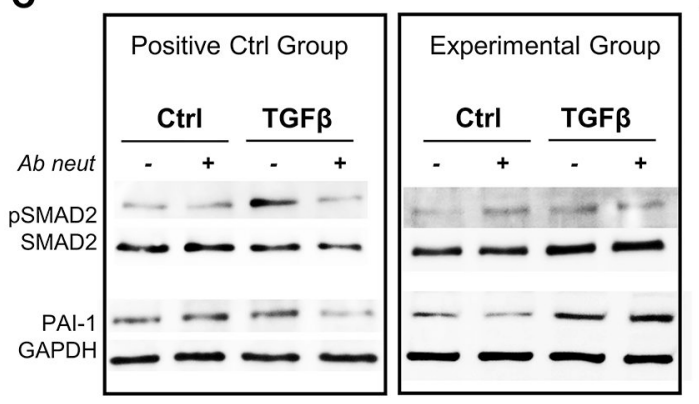

B

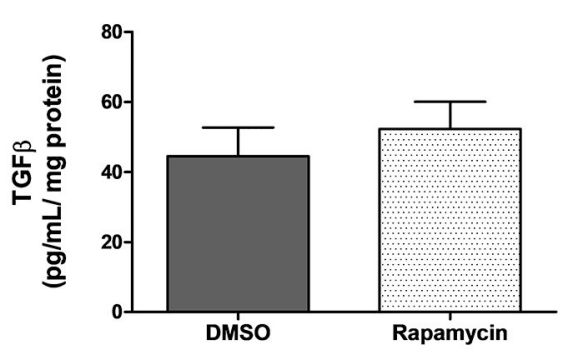

D

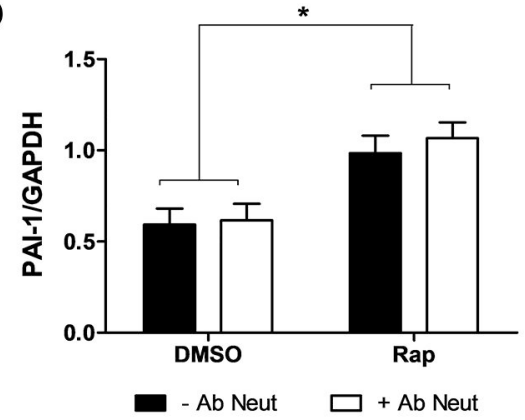

Figure 3.

Rapamycin induces TGFRI pathway independent of TGF $\beta$. Active TGF $\beta$ release after rapamycin treatment for (A) 30 minutes $(n=6)$ or $(\mathbf{B}) 24$ hours $(n=14)$. (C) Western blot for SMAD2 phosphorylation and PAI-1 expression after neutralizing TGF $\beta$ experiments. The efficiency of neutralization was demonstrated in the positive control group where pSMAD2 and PAI-1 induction by TGF $\beta$ was blocked by the neutralizing TGF $\beta$ antibody (D) PAI-1 expression after 24 hours of rapamycin treatment in the presence of neutralizing TGF $\beta$. Each bar represents mean \pm SEM of 7 independent experiments. * indicates $p<0.05$. 

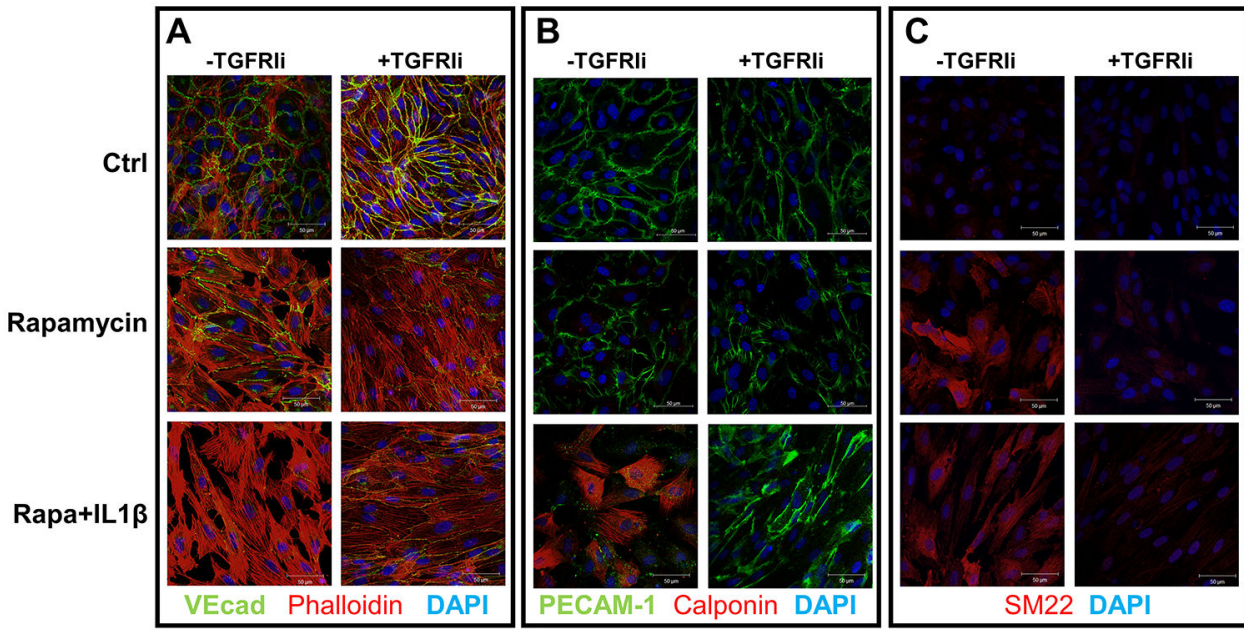

D

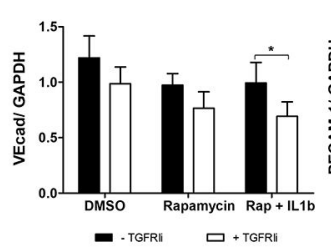

$\mathbf{F}$
G
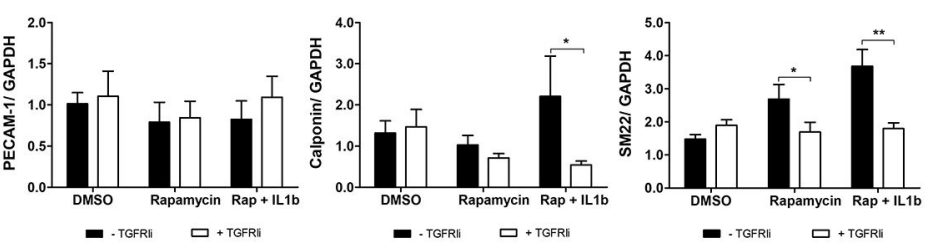

Figure 4.

Rapamycin induces Endothelial-to-Mesenchymal Transition, which is potentiated in the presence of IL-1 $\beta$. (A - C) Immunocytochemistry of EndMT markers after rapamycin treatment for 96 hours in the presence or absence of TGFRI inhibitor. Endothelial markers in green, mesenchymal markers in red, nuclei in blue (DAPI). (A) Double staining for VEcadherin and Phalloidin; (B) double staining for PECAM-1 and Calponin; (C) SM22. Each image is representative of 3 independent experiments. (D-G) Western blot for EndMT markers: (D) VE-Cadherin, N=7; (E) PECAM-1, N=4; (F) Calponin, N=8; (G) SM22, N=5. Each bar represents mean \pm SEM. * indicates $\mathrm{p}<0.05$ and $* * \mathrm{p}<0.001$. 
A
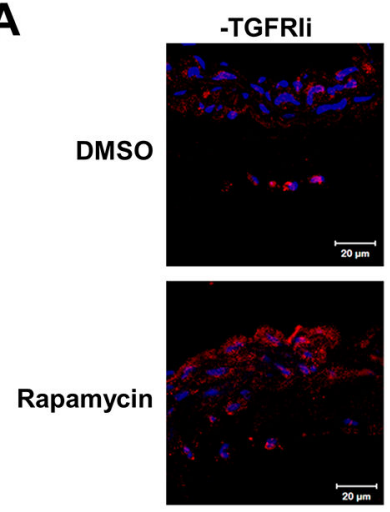

C

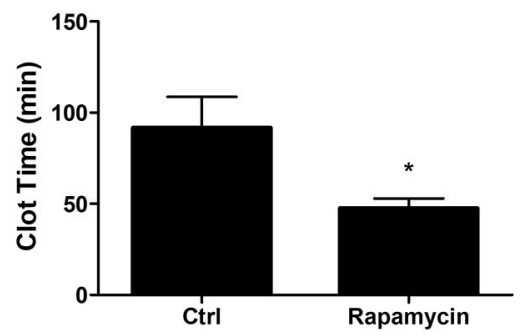

B
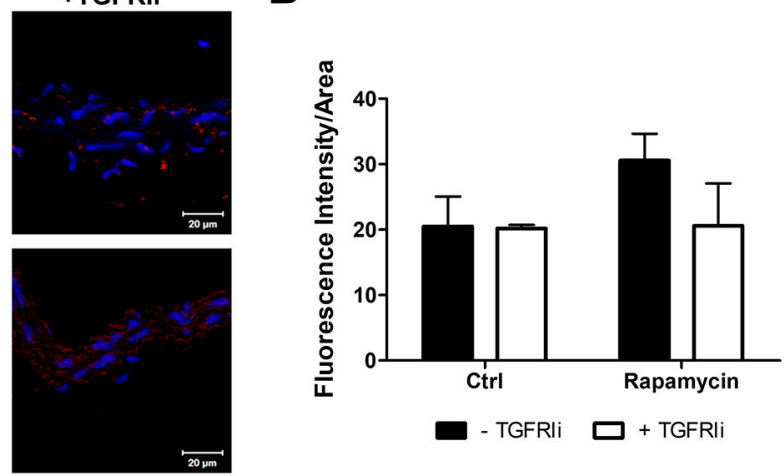

D

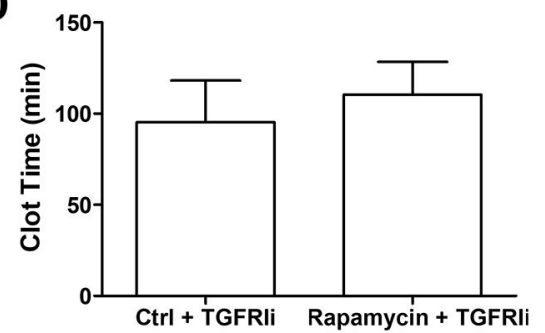

Figure 5.

Blockage of TGFRI activation decreases rapamycin-induced PAI-1 expression and thrombogenicity in vivo. (A) PAI-1 immunohistochemistry in carotid artery of mouse treated with rapamycin and/or TGFRIi. Confocal microscopy images at 400× original magnification. (B) PAI-1 immunohistochemistry fluorescence intensity quantification. Each bar represents mean \pm SEM of 3-5 animals. Photochemical thrombosis injury in carotid artery of mouse treated with $(\mathbf{C})$ rapamycin without TGFRIi or (D) in the presence of TGFRIi. (C) Thrombosis in rapamycin-treated mice occurred faster than in non-treated mice. (D) TGFRI inhibitor blocked the prothrombotic effects of rapamycin. Each bar represents mean \pm SEM of $3-6$ animals. * indicates $p<0.05$. 